\title{
Three-dimensional flow in open channels
}

\author{
BY Frank ENGELUND,
}

PROFESSOR AT THE POLXTEKNISK LIEREANSTALT, JANMARKS TEKNISKE HOISKOLE, COPLNHAGEN

\begin{abstract}
The paper deals with the deduction of a general equation for the shape of the free water table in open channel flow, taking account of non-hydrostatic pressure distribution and gradual irregularities of the bottom. It may be considered an extension to three dimensions of the classical Boussinesq-theory for gradually varied flow.
\end{abstract}

In a previous paper [1] in this journal ( $L a$ Houille Blanche, No. 4, 1953), written in collaboration with Mr. J. Munch-Petersen, steady flows in contracted and expanded channels were discussed by means of potential theory, and the results compared with experiments.

The main results may be summarized in the following way:

The older theory of flow through channel expansions, based on an analogy with supersonic flow in compressible gases, is satisfactory only for high-velocity flow and only when the channel width is great compared with the water depth.

The previous paper showed that much better agreement hetween theory and experiments was obtained from the application of potential theory. This was considered remarliable, since potential theory assumes a negligible loss of energy, but it was proved that the apparent shock-fronts actually might be accounted for by the superposition of a series of double-periodic functions satisfying Laplace's equation. of course potential theory could not be valid if considerable surface rollers were formed.

In this paper an attempt is made to deduce an explicit differential equation for the water depth $y_{0}$ in a three-dimension gradually varied flow in broad rectangular channels. Before entering this problem some remarks concerning the corresponding two-dimensional problem are necessary.
In this case, the differential equation describing the variation of water depth reads :

$$
\begin{aligned}
\mathrm{I}_{b}-\mathrm{I}=\frac{d s}{d x} & +\left(1-\mathscr{F}^{2}\right) \frac{d y_{10}}{d x} \\
& +\frac{v_{m b}^{2}}{3 g} y_{0} \frac{d^{3} y_{0}}{d x^{3}}+\frac{v_{m^{2}}{ }^{3} g}{2 g} y_{0} \frac{d^{3} s}{d x^{3}}
\end{aligned}
$$

assuming moderate slope of the bed.

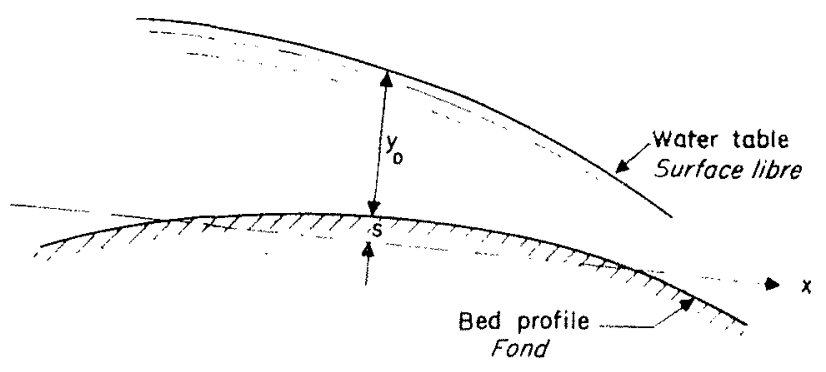

The $x$-axis is chosen as an "avernge bed," the deviation of the actual bed from this being denoted by $s$ and counted positive when the actual bed lies above the $x$-axis. $I_{b}$ is the (moderate) slope of the $x$-axis, while $I$ is the hydraulic gradient (i.e. the slope of the energy-line). $y_{0}$ denotes the depth, $v_{m}$ the average velocily, $g$ the acceleration of gravity and $\mathscr{g}\left(=v_{m} / \sqrt{ } g_{y_{0}}\right)$ the Froude number. Ec. (1) has originally been given by Boussinesq.

The most direct way of ehecking the validity of (1) is to compare solutions with results obtain- 
ed by more exact methods, such as potential theory. For example it is well known, that equation (1) when applied to the solitary wave, yields a very accurate solution.

As a further example consider the flow of a frictionless fluid in a horizontal channel with regular bed ondulations. In this case it is usually assumed that:

$$
\mathrm{I}_{b}=0 \quad \text { and } s=s_{0} \sin \frac{2 \pi x}{\lambda}
$$

in which $s_{0}$ is the amplitude and $\lambda$ the wave length of the bed ondulations, It is easily seen that the solution of Eq. (1) is given by an expression of the form:

$$
y_{0}=\text { const. }+a \sin \frac{2 \pi x}{\lambda}
$$

and that:

$$
\begin{aligned}
\frac{s_{0}}{a+s_{0}} & =\frac{1+\frac{1}{3}\left(\frac{2 \pi y_{0}}{\lambda}\right)^{2}}{1-\frac{1}{6}\left(\frac{2 \pi y_{0}}{\lambda}\right)^{2}} \\
& -\frac{g \lambda}{2 \pi v^{2}} \cdot \frac{\frac{2 \pi y_{0}}{\lambda}}{1-\frac{1}{6}\left(\frac{2 \pi y_{0}}{\lambda}\right)^{2}}
\end{aligned}
$$

The first order solution, as obtained from potential theory, is, correspondingly,

$$
\frac{s_{0}}{\alpha+s_{0}}=\cosh \left(\frac{2 \pi y_{0}}{\lambda}\right)-\frac{g \lambda}{2 \pi \nu^{2}} \sin \left(\frac{2 \pi y_{0}}{\lambda}\right)
$$

(see f.i. [2] p. 409).

Now it is easy to verify that the solutions agree within a margin of approximately 3 per cent, when the parameter $2 \pi y_{0} / \lambda$ is less than unity, i.e. for long waves.

When curvature is negligible, the last two terms of Eq. (1) vanish, and Eq. (1) reduces to the ordinary equation used for calculations of gradually varied flow in channels.

Turning now to the problem of three-dimensional flow, we consider the flow in a broad rectangular channel. The $x$-axis is chosen in the direction of flow (over the "average bed"), the $z$-axis is horizontal and perpendicular to the side-walls of the channel, while the $y$-axis is perpendicular to the $x$-z-plane (positive upwards). The slope of the plane "average bed" is $I_{m}$ while the height of the actual bed above the average bed is denoted by $s$.

Under this assumption we have

$$
s=s(x, z)
$$

where $s$ and its derivatives are assumed to be small quantities.
The equation of motion for an arbitrary unit cube following the fluid becomes:

$$
\frac{d \bar{v}}{d t}=-\operatorname{grad}\left(\frac{p}{\rho}+g h\right)+\frac{\bar{s}}{\rho}
$$

in which $\rho$ is the density, $\bar{v}$ the velocity vector, $p$ the pressure, $h$ the heigth of the cube above some fixed level and $\bar{s}$ the resulting shear force on the cube.

When the flow is steady we have:

$$
\frac{d \bar{v}}{d t}=\frac{1}{2} \operatorname{grad} v^{2}-\bar{v} \times \operatorname{curl} \bar{v}
$$

where $X$ denotes the vector product.

Substitution into Eq. (2) yields:

$$
\operatorname{grad}\left(\frac{p}{\rho}+g h+\frac{1}{2} v^{2}\right)=\bar{v} \times \operatorname{curl} \bar{v}+\frac{\bar{s}}{\rho}
$$

Now it is assumed that the flow may be considered as a slight perturbation of a linear flow. Hence the velocity components $v_{y}$ and $v_{z}$ are small compared with $v_{x}$, so that second order terms in the quantities may be neglected. Similarly, all partial derivatives of the velocity components are assumed small and all derivatives of the shear stress are neglected except $\partial \tau / \partial y$.

Under these assumptions Eq. (3), when written in the above mentioned coordinates, become:

$$
\begin{aligned}
& \frac{\partial}{\partial x}\left(g h+\frac{p}{\rho}+\frac{1}{2} v^{2}\right)=\frac{1}{\rho} \frac{\partial \tau}{\partial y} \\
& \frac{\partial}{\partial y}\left(g h+\frac{p}{\rho}+\frac{1}{2} v^{2}\right)=v_{x}\left(\frac{\partial v_{x}}{\partial y}-\frac{\partial v_{y}}{\partial x}\right) \\
& \frac{\partial}{\partial z}\left(g h+\frac{p}{\rho}+\frac{1}{2} v^{2}\right)=v_{x}\left(\frac{\partial v_{x}}{\partial z}-\frac{\partial v_{z}}{\partial x}\right)
\end{aligned}
$$

Besides the equations of motion we have the equation of continuity:

$$
\operatorname{div} \bar{v}=\frac{\partial v_{x}}{\partial x}+\frac{\partial v_{y}}{\partial y}+\frac{\partial v_{z}}{\partial z}=0,
$$

as the fluid is incompressible.

The last basic assumption is that the velocity component $v_{y}$ varies linearly with the height $(y-s)$ above the bed:

$$
v_{y}=\left[\frac{\partial s}{\partial x}+\frac{y-s}{y_{0}} \cdot \frac{\partial y_{0}}{\partial x}\right] v_{x}
$$

from which:

$$
\frac{\partial v_{y}}{\partial x}=\left[\frac{\partial^{2} s}{\partial x^{2}}+\frac{y-s}{y_{0}} \cdot \frac{\partial^{2} y_{0}}{\partial x^{2}}\right] v_{x}
$$

second order terms being neglected. In Eq. (6) the condition that $y=s$ corresponds to the bed surface, while $y=y_{0}+s$ corresponds to the water surface, $y_{0}$ being the depth. 
Eq. $(4 b)$ is now written as :

$$
\frac{\partial}{\partial y}\left[g h+\frac{p}{p}\right]=--v_{x} \frac{\partial v_{u}}{\partial x}
$$

the difference between $v^{2}$ and $v_{x}{ }^{2}$ being of second order.

Substitution of Eq. $(6 a)$ then gives.

$$
\frac{\partial}{\partial y}\left[g h+\frac{p}{\rho}\right]=-v^{2}\left[\frac{\partial^{2} s}{\partial x^{2}}+\frac{y-s}{J_{0}} \frac{\partial^{2} y_{0}}{\partial x^{2}}\right]
$$

This equation may be integrated between the boundaries $s$ and $y$ along a line corresponding to fixed values of $x$ and $z$ :

$$
\begin{aligned}
g h+\frac{p}{\rho}= & -\frac{\partial^{2} s}{\partial x^{2}} \int_{s}^{y} v^{2} d y \\
& -\frac{1}{y_{0}} \frac{\partial^{2} y_{0}}{\partial x^{2}} \int_{8}^{y} v^{2}(y-s) d y+\text { const. }
\end{aligned}
$$

Taking $v$ to be constant along the line we introduce an error which may be cancelled out by the introduction of velocity-coefficients in the final expressions. As these coefficients depart only slightly from unity they are usually not introduced at all.

Hence, the above integration gives us an expression for the distribution of the pressure:

$$
\begin{aligned}
g h+\frac{p}{\rho}=-v^{2} & (y-s) \frac{\partial^{2} s}{\partial x^{2}} \\
& -\frac{1}{2} v^{2} \frac{y^{2}-2 s y}{y_{0}} \frac{\partial^{2} y_{0}}{\partial x^{2}}+\text { const. }
\end{aligned}
$$

Introducing the boundary conditions,

$$
p=0 \text { at } y=y_{0}+s,
$$

we arrive at:

$$
\begin{aligned}
g\left(h-h_{0}\right)+\frac{p}{\rho}= & v^{2}\left(y_{0}-y\right) \frac{\partial^{2} s}{\partial x^{2}} \\
& +\frac{1}{2} v^{2}\left(y_{0}-\frac{y^{2}}{y_{0}}\right) \frac{\partial^{2} y_{0}}{\partial x^{2}}
\end{aligned}
$$

in which $h_{0}$ corresponds to the height of the water table along the considered line. Second power terms are neglected.

From this expression we are able to calculate the integral:

$$
\int_{s}^{y_{0}+s} \frac{\partial}{\partial x}\left(g h+\frac{p}{\rho}\right) d y
$$

To this end we substitute:

$$
h_{0}=h_{b}+s+y_{0}
$$

where $h_{b}$ indicates the level of the "average bed." By differentiation we obtain:

$\frac{\partial h_{0}}{\partial x}=\frac{\partial h_{b}}{\partial x}+\frac{\partial s}{\partial x}+\frac{\partial y_{n}}{\partial x}=\div \mathrm{I}_{m}+\frac{\partial s}{\partial x}+\frac{\partial y_{0}}{\partial x}$
Then, from Eq. (7) we have:

$$
\begin{aligned}
\int_{s}^{s+y_{0}} \frac{\partial}{\partial x}\left(g h+\frac{p}{\rho}\right) d y & \\
=g y_{0}\left(-\mathrm{I}_{m}+\frac{\partial s}{\partial x}+\frac{\partial y_{0}}{\partial x}\right) & +\frac{1}{2} v^{2} y_{0}{ }^{2} \frac{\partial^{3} s}{\partial x^{3}} \\
& +\frac{1}{3} v^{2} y_{0}^{2} \frac{\partial^{3} y_{0}}{\partial x^{3}}
\end{aligned}
$$

Assuming, as above, that the velocity is approximately constant from the bed to the surface we have that:

$$
\int_{s}^{s+\psi_{0}} \frac{1}{2} \frac{\partial v^{2}}{\partial x} d y=v y_{0} \frac{\partial v}{\partial x}
$$

Integrating Eq. (4a) between the boundaries $s$ and $s+y_{0}$ yields:

$$
\begin{aligned}
-\frac{\tau_{0}}{\rho}= & g y_{0} \\
\dot{\times}\left[-I_{m}\right. & +\frac{\partial s}{\partial x}+\frac{\partial y_{0}}{\partial x}+\frac{1}{2} \frac{v^{2}}{g} y_{0} \frac{\partial^{3} s}{\partial x^{3}} \\
& \left.+\frac{1}{3} \frac{v^{2}}{g} y_{0} \frac{\partial^{3} y_{0}}{\partial x^{3}}+\frac{v}{g} \frac{\partial v}{\partial x}\right]
\end{aligned}
$$

and, putting $\tau_{0}=\gamma y_{0} \mathrm{I}$,

$$
\begin{aligned}
\mathrm{I}_{m}-\mathrm{I}= & \frac{\partial s}{\partial x}+\frac{\partial y_{0}}{\partial x}+\frac{v}{g} \frac{\partial y}{\partial x} \\
& +\frac{1}{2} \frac{v^{2}}{g} y_{0} \frac{\partial^{3} s}{\partial x^{3}}+\frac{1}{3} \frac{v_{0}^{2}}{g} y_{0} \frac{\partial^{3} y_{0}}{\partial x^{3}}
\end{aligned}
$$

At first sight Eq. (8) is equivalent to Eq. (1) but in fact it differs from that equation in that, in Eq. (8), $v$ and I vary in the direction of the $z$-axis. In order to take this variation into account, we proceed to integrate Eq. (4t) between $y=s$ and $y=y_{0}+s$.

The application of Eq. (7) yields:

$$
\begin{array}{r}
\int_{s}^{3+y_{0}} \frac{\partial}{\partial z}\left(g h+\frac{p}{\rho}\right) d y=g y_{0}\left(\frac{\partial s}{\partial z}+\frac{\partial y_{0}}{\partial z}\right) \\
+-\frac{1}{2} v^{2} y_{0}^{2} \frac{\partial^{3} s}{\partial z \partial x^{2}}+\frac{1}{3} b^{2} y_{0}^{2} \frac{\partial^{3} y_{0}}{\partial z \partial x^{2}}
\end{array}
$$

Since the last term on the Ieft hand side of Eq. $(4 c)$ is then cancelled out by the first term on the right hand side, Eq. (9) is equal to :

$$
\begin{aligned}
-\int_{s}^{s+y_{0}} v_{s} \frac{\partial v_{z}}{\partial x} d y= & -v y_{0} \frac{\partial v_{z}}{\partial x}, \text { or } \\
-\frac{v}{g} \cdot \frac{\partial v_{z}}{\partial x}=\frac{\partial s}{\partial z} & +\frac{\partial y_{01}}{\partial z}+\frac{1}{2} \frac{v^{2} y_{0}}{g} \frac{\partial^{3} s}{\partial z \partial x^{2}} \\
& +\frac{1}{3} \frac{v^{2} y_{0}}{g} \frac{\partial^{3} y_{0}}{\partial z \partial x^{2}}
\end{aligned}
$$


An expression for the left hand side of this equation is obtained in the following way. From Eq. (6) :

$$
\frac{\partial v_{y}}{\partial y}=\frac{v}{y_{0}} \cdot \frac{\partial y_{0}}{\partial x}
$$

Then from Eq. (5):

$$
\frac{\partial v_{z}}{\partial z}=-\frac{\partial v}{\partial x}-\frac{v}{y_{0}} \frac{\partial y_{0}}{\partial x}
$$

from which:

$$
\frac{\partial^{2} v_{z}}{\partial x \partial z}=-\frac{\partial^{2} v}{\partial x^{2}}-\frac{v}{y_{0}} \frac{\partial^{2} y_{0}}{\partial x^{2}}
$$

When Eq. (10) is differentiated with respect to $z$, this gives:

$$
\begin{aligned}
-\frac{v}{g} \frac{\partial^{2} v_{z}}{\partial x \partial z}= & +\frac{v}{g}\left(\frac{\partial^{2} v}{\partial x^{2}}+\frac{v}{y_{0}} \frac{\partial^{2} y_{0}}{\partial x^{2}}\right) \\
= & +\frac{v}{g} \frac{\partial^{2} v}{\partial x^{2}}+g^{2} \frac{\partial^{2} y_{0}}{\partial x^{2}} \\
= & \frac{\partial^{2} s}{\partial z^{2}}+\frac{\partial^{2} y_{0}}{\partial z^{2}}+\frac{1}{2} \frac{v^{2} y_{0}}{g} \frac{\partial^{4} s}{\partial x^{2} \partial z^{2}} \\
& +\frac{1}{3} \frac{v^{2} y_{0}}{g} \frac{\partial^{4} y_{0}}{\partial x^{2} \partial z^{2}}
\end{aligned}
$$

Finally, if Eq. (8) is differentiated with respect to $x$ and Eq. (11) is substituted, we get the fundamental differential equation for the threedimensional flow:

$$
\begin{gathered}
-\frac{\partial \mathrm{I}}{\partial x}=\nabla^{2} s+\nabla^{2} y_{0}-\mathfrak{F}^{2} \frac{\partial^{2} y_{0}}{\partial x^{2}}+\frac{1}{2} \mathfrak{F}^{2} y_{\vartheta^{2}} \\
\times \frac{\partial^{2}}{\partial x^{2}}\left(\nabla^{2} \mathrm{~s}\right)+\frac{1}{3} \mathfrak{F}^{2} y_{0}{ }^{2} \frac{\partial^{2}}{\partial x^{2}}\left(\nabla^{2} y_{0}\right)
\end{gathered}
$$

where:

$$
\nabla^{2}=\frac{\partial^{2}}{\partial x^{2}}+\frac{\partial^{2}}{\partial z^{2}}
$$

is the two-dimensional form of the Laplacian operator.

It should be noted that equation (1) is the one dimension from of Eq. (12).

In order to form rigorous solutions of equation
(12) we proceed to consider the doubly periodic flow of a frictionless fluid in a rectangular channel with a plane and horizontal bed. This example was treated by first order potential theory in Ref. [1].

The water surface is assumed to be given by the expression:

$$
y_{0}=y_{m}+\alpha \sin \frac{2 \pi x}{\lambda} \cos \frac{2 \pi z}{b}
$$

in which $y_{m}$ is the average depth, $a$ the amplitude, $\lambda$ the wave length and $b$ the width of the channel. Considering the above stated simplifications, Eq. (12) reduces to:

$\nabla^{2} y_{0}-\mathscr{F}^{2} \frac{\partial^{2} y_{0}}{\partial x^{2}}+\frac{1}{3} \mathscr{F}^{2} y_{m}{ }^{2} \frac{\partial^{2}}{\partial x^{2}}\left(\nabla^{2} y_{0}\right)=0$

When (13) is substituted into Eq. (14) we obtain, after reduction:

$$
\mathscr{F}^{2}\left(\frac{2 \pi y_{m}}{\lambda}\right)^{2}=\frac{A^{2}}{1+(1 / 3) A^{2}}
$$

in which:

$$
\mathrm{A}=\sqrt{\left(\frac{2 \pi y_{\mathrm{m}}}{\lambda}\right)^{2}+\left(\frac{2 \pi y_{\mathrm{m}_{1}}}{b}\right)^{2}}
$$

The corresponding relation deduced from potential theory (1) is:

$$
\mathscr{g}^{2}\left(\frac{2 \pi y_{\mathrm{m}}}{\lambda}\right)^{2}=\mathrm{A} \tanh \mathrm{A}
$$

where A still is given by Eq. (15 a) .

$\mathrm{Eq}$. (15) is a close approximation to the more correct Eq. (16) provided that $A<1$. Thus the validity of Eq. (12) is confirmed in the case of a small curvation of the water surface.

\section{Références}

[1] Frank Engelund and Johs. Mungh-Petensen. Steady flow in contracted and expanded rectangular channels. La Houille Blanche, $\mathrm{n}^{\circ} 4$, aoûtseptembre 1953.

[2] H. LAMB. -- Hydrodynamics, sixth edition, 1945. 


\title{
Écoulement à surface libre à trois dimensions
}

\author{
PAR F. ENGELUND
}

L'auteur étudie dans cet article l'écoulement permanent varié en canal rectangulaire de largeur constante importante par rapport à la profondeur. Le fond du canal est une surface donnée par sa surélévation $s(x, y)$ au-dessus d'un fond plan moyen de pente $\mathrm{I}_{b}$ dans la direction $x$.

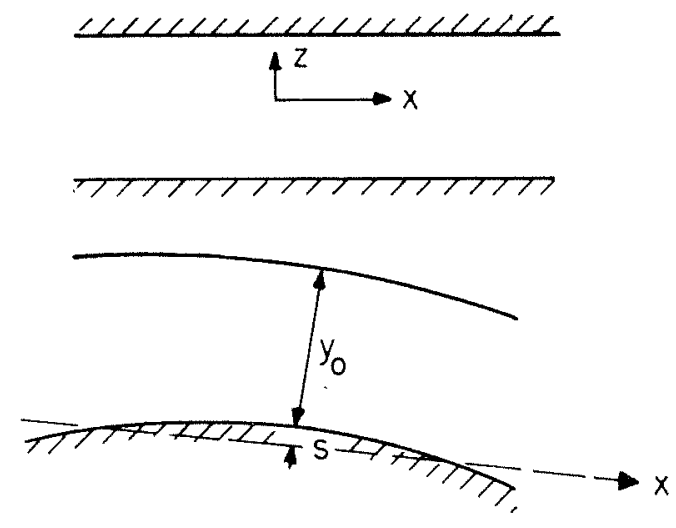

L'écoulement considéré est caractérisé par le fait qu'il diffère peu de l'écoulement général dans la direction $x$, ce qui amène l'auteur à poser les hypothèses simplificatrices correspondantes et le conduit finalement à l'équation (12), qui caractérise l'écoulement à trois dimensions étudié. (Dans cette équation, I est la pente de la ligne de charge et $\mathscr{G}_{t}=v_{m} / \sqrt{g y_{0}}$ le nombre de Froude correspondant à l'écoulement moyen.)

Pour la clarté de l'exposé, le cas de l'écoulement à deux dimensions est présenté au début de l'article (équation 1). L'exemple de l'écoulement sans frottement sur un fond ondulé est traité au moyen de cette équation. Les résultats obtenus sont très voisins dans ce cas de la solution au premier ordre donnée par la théorie du potentiel.

L'équation (12) est utilisée, à titre d'exemple, pour traiter on fluide parfait le cas de l'écoulement doublement périodique dans un canal rectangulaire à fond plan horizontal. La solution en est très proche de celle que l'auteur avait donnée, en collaboration avec J. Munch Petersen (ref. [1]), en utilisant la théorie du potentiel au premier ordre. 